\title{
Publisher's Note: Large fraction of crystal directions leads to ion channeling [Phys. Rev. B 94, 214109 (2016)]
}

K. Nordlund, F. Djurabekova, and G. Hobler

(Received 23 February 2017; published 8 March 2017)

DOI: 10.1103/PhysRevB.95.099901

This paper was published online on 16 December 2016 with an incorrect author affiliation. The affiliation of F. Djurabekova should read as "Helsinki Institute of Physics and Department of Physics, P.O. Box 43, FIN-00014 University of Helsinki, Finland." The author affiliation has been corrected as of 23 February 2017. The author affiliation is incorrect in the printed version of the journal. 